\title{
Prevenção e detecção do câncer bucal: planejamento participativo como estratégia para ampliação da cobertura populacional em idosos
}

\author{
Prevention and detection of oral cancer: participatory planning \\ as a strategy to broaden coverage in the elderly population
}

Jaqueline Vilela Bulgareli ${ }^{1}$

Olívia Cristina Caseto Furian Diniz ${ }^{2}$

Eduardo Tanajura de Faria ${ }^{2}$

Fabiana de Lima Vazquez ${ }^{1}$

Karine Laura Cortellazzi ${ }^{1}$

Antonio Carlos Pereira ${ }^{1}$

${ }^{1}$ Faculdade de Odontologia de Piracicaba, Unicamp. Av. Limeira, 901 Areião. 13.414-018 Piracicaba SP Brasil.pmsb.jaqueline@ yahoo.com.br

${ }^{2}$ Secretaria Municipal de Saúde de Marília (SP).

\begin{abstract}
With respect to addressing oral cancer as a public health problem and the need to conduct early diagnosis to ensure a favorable prognosis for patients, the city of Marilia in the state of São Paulo stages an annual campaign for prevention and early detection of oral cancer. This study sets out to evaluate the participatory planning of the teams at health facilities, seeking to list the difficulties encountered and strategies adopted over a five-year period (2006-2011) by analyzing the coverage of oral examinations for prevention and detection of disease. Strategies for tackling difficulties, issues of epidemiological coordination and surveillance raised by dentists, generated an increase in the coverage of tests performed in the population (> 60 years) from $21 \%$ in 2006 to $62 \%$ in 2011. The conclusion is that the development of strategies with the participation of health teams produces quite encouraging results, ensuring that the lesions of oral cancer are diagnosed and treated early from the perspective of a more favorable prognosis.
\end{abstract}

Key words Carcinoma, Oral neoplasm, Primary health care
Resumo Considerando a abordagem do câncer bucal um problema de saúde pública e a necessidade de fortalecer o diagnóstico precoce para garantir um prognóstico favorável aos pacientes, $o$ município de Marília (SP) realiza anualmente uma ação de prevenção e detecção precoce do câncer bucal. Este estudo teve por objetivo avaliar o planejamento participativo das equipes das unidades de saúde, buscando elencar as dificuldades encontradas e as estratégias adotadas no período de cinco anos (2006-2011), através da análise da cobertura de exames bucais de prevenção e detecção da doença. As estratégias para enfrentamento das dificuldades, aspectos de coordenação e vigilância epidemiológica levantadas pelos cirurgiõesdentistas, geraram um incremento na cobertura de exames realizados na população (> 60 anos) passando de uma cobertura de 21\% em 2006 para $62 \%$ em 2011. Conclui-se que o desenvolvimento das estratégias com participação das equipes de saúde vem repercutindo em resultados bastante satisfatórios, garantindo que as lesões de câncer bucal sejam diagnosticadas e tratadas precocemente sob a ótica de um prognóstico mais favorável. Palavras-chave Carcinoma, Neoplasia bucais, Prevenção primária 


\section{Introdução}

O câncer atinge milhões de pessoas no mundo, independente de classe social, cultura ou religião, o impacto do diagnóstico é geralmente aterrador, pois apesar dos avanços terapêuticos, ainda permanece o estigma de doença dolorosa, incapacitante, mutilante e, por vezes, mortal. Assim, apesar dos recentes avanços no diagnóstico e tratamento da doença, que asseguram a remissão e possível cura, o câncer permanece como uma doença relacionada com a desesperança, a dor, o medo e a morte ${ }^{1}$.

Segundo o Instituto Nacional de Câncer ${ }^{2}$, Câncer é o nome dado a um conjunto de mais de 100 doenças que têm em comum o crescimento desordenado (maligno) de células que invadem os tecidos e órgãos, podendo espalhar-se (metástase) para outras regiões do corpo. Conforme a Organização Mundial da Saúde ${ }^{3}$, esta atinge cerca de 12,4 milhões de pessoas e ocasiona 7,6 milhões de mortes por ano no mundo.

No Brasil, o câncer é a segunda causa de morte por doença, sendo, portanto, indubitavelmente um problema de saúde pública ${ }^{4}$. As estimativas do INCA apontam para a incidência de 518.510 novos casos de câncer no ano de 2012 no país. Das diversas causas de morte no mundo, o câncer é a única que continua a crescer independente do país ou continente e, nos países em desenvolvimento, é atualmente responsável por uma entre dez mortes ${ }^{3}$.

Compreende-se como câncer bucal o grupo de tumores classificados pela Classificação Internacional de Doenças - CID-10 como: Neoplasia maligna da gengiva, Neoplasia maligna do assoalho da boca, Neoplasia maligna do palato, $\mathrm{Ne}$ oplasia maligna de outras partes e de partes não especificadas da boca. O câncer bucal no Brasil, conforme a estimativa de incidência do INCA para o ano de 2012, acometerá 9.990 homens o que representa a quinta localização anatômica no sexo masculino, além de 4.180 mulheres ${ }^{5}$.

A etiologia dessa neoplasia é multifatorial e, apesar de todo o avanço tecnológico obtido até o momento, os agentes etiológicos para o câncer ainda são uma incógnita. São considerados fatores de risco extrínsecos para o carcinoma bucal as substâncias químicas (tabaco, álcool), agentes físicos (traumas mecânicos) e biológicos e os intrínsecos são aqueles que correspondem aos estados sistêmicos do indivíduo ${ }^{6}$.

Como fatores de proteção, alguns estudos observaram que o consumo de frutas e de vegetais consiste em medidas efetivas contra o Cân- cer Bucal ${ }^{7-9}$. Assim, Gomes et al. ${ }^{10}$ afirmaram que a prevenção se volta para uma ação orientada para que o sujeito não adoeça e possa desfrutar de melhor qualidade de vida; para tal, é necessário envolvê-lo com informações relevantes para que se insira ativamente e possa incorporar hábitos preventivos.

A mortalidade por câncer bucal corresponde a pouco menos de $30 \%$ dos casos novos, o que significa um prognóstico razoável. A sobrevida média estimada mundial em cinco anos é cerca de $46 \%{ }^{11}$.

A grande maioria das neoplasias malignas da boca e complexo maxilomandibular é constituída por carcinomas epidermoides, atingindo $90 \%$ dos casos, e o restante é representado por sarcomas, melanomas e tumores malignos de glândulas salivares. O carcinoma epidermoide representa, portanto, a condição mais séria entre as neoplasias que acometem a cavidade bucal, levando à morte grande parcela dos pacientes, pois a desinformação contribui para postergar a procura por um serviço de saúde ou um serviço especializado. O diagnóstico tardio pode estar relacionado a diversos fatores, dentre eles: a desinformação da população, a falta de alerta dos profissionais da saúde para o diagnóstico precoce dos casos; e a falta de rotinas abrangentes programadas nos serviços de saúde, públicos e privados, que favoreçam a detecção do câncer ${ }^{12}$.

Considerando que o câncer bucal tem que ser abordado como um problema de saúde pública, além da necessidade de fortalecer o diagnóstico precoce para garantir um prognóstico favorável aos pacientes acometidos pela doença, o município de Marília (SP) realiza anualmente uma ação de prevenção e detecção precoce do câncer bucal, com o apoio da Fundação Oncocentro de São Paulo (FOSP) $)^{13}$, a qual foi incentivadora para que as ações de detecção do câncer bucal fossem intensificadas juntamente à Campanha Nacional de Vacinação do Idoso, tendo como objetivo examinar o maior número de idosos, uma vez que a incidência do câncer bucal é maior a partir da $5^{\text {a }}$ década de vida.

A ação de prevenção e detecção do câncer bucal, em Marília, é realizada desde 1996, envolvendo todas as unidades de saúde vinculadas ao SUS além de pontos estratégicos, sendo que a partir do ano de 1999, houve a sua associação com a campanha de vacinação contra a influenza em idosos, sendo realizadas diversas ações como: capacitações anuais dos cirurgiões dentistas (CD) envolvidos, divulgação na mídia e palestras em unidades de saúde (grupos de $3^{\mathrm{a}}$ ida- 
de). Até o ano de 2004, os exames eram realizados somente no sábado da abertura da campanha de vacinação, sendo que a meta era examinar todos os idosos vacinados.

Além do sábado de abertura, em 2005, a ação se estendia à semana subsequente, destinando um período do dia dos dentistas envolvidos para a ação. Os Agentes Comunitários de Saúde, Auxiliar de Consultório Dentário, CD e equipe de saúde divulgavam na comunidade os períodos para realização do exame.

Ao longo dos anos a cobertura da vacinação sempre foi notoriamente superior à cobertura do exame bucal para detecção do câncer na cavidade oral, quando no ano de 2006 houve a integração da Coordenação de Saúde Bucal e a Vigilância Epidemiológica do município com a elaboração de um plano operativo conjunto, com o objetivo de aproximar as coberturas das campanhas de vacinação e do câncer bucal.

Em 2007, a ação de prevenção do câncer bucal acompanhou a de vacinação, se estendendo por duas semanas, com a agenda direcionada para os exames bucais. A meta era examinar 100\% dos idosos do município, conforme preconizado pela FOSP.

Algumas ações foram incrementadas em 2008, como a gestão participativa para pactuar as metas e as estratégias para enfrentamento das dificuldades encontradas, principalmente em relação ao alcance da meta proposta. Para tanto a coordenação de saúde bucal organizou reunião de planejamento com os CD da rede de atenção básica, na qual a meta foi sugerida pelas próprias equipes das unidades de saúde, estimulando-as a refletir a prática de acordo com a realidade local.

Neste mesmo ano, os CD das unidades de saúde com menor número de idosos em sua área de abrangência e os CD substitutos, ambos da Estratégia Saúde da Família (ESF), foram remanejados para as Unidades Básicas de Saúde (UBS) para auxílio na realização dos exames bucais nas unidades (inclusive nos horários de almoço) e em visitas domiciliares (VD) para os idosos acamados, visto que é notório o grande número de idosos das UBS, além daqueles que procuram estas unidades sem pertencer ao território de abrangência das mesmas, o que é considerado “invasão”.

No ano de 2009 mantiveram-se as estratégias do ano anterior, acrescentando a realização de exames bucais em supermercados e feiras livres, acompanhando os postos volantes da campanha de vacinação e computou-se o número de pacientes que não permitiram o exame bucal - recusas.
Considerando o número significativo de recusas registradas no ano anterior, em 2010 na reunião de planejamento da ação, foram abordadas as causas e as propostas das ações para minimizar este resultado. Além disso, incluiu-se o setor do comércio, representado por estabelecimentos comerciais populares da cidade com grande fluxo de pessoas, como mais um ponto estratégico, volante, na ação externa e estendeuse a capacitação para os demais profissionais das unidades de saúde (médicos, enfermeiras e fonoaudiólogas).

\section{Objetivo}

Este estudo teve por objetivo avaliar o planejamento participativo das equipes das unidades de saúde, buscando elencar as dificuldades encontradas e descrever as estratégias adotadas para o desenvolvimento e melhoria contínua da ação, no período de cinco anos (2006-2011), através da análise da cobertura de exames bucais de prevenção e detecção da doença.

\section{Materiais e Método}

A ação de prevenção e detecção precoce do câncer bucal realizada pela Secretaria Municipal da Saúde em Marília acontece anualmente, associada à Campanha de Vacinação dos Idosos, tem como foco a identificação precoce da doença, além do estimulo ao autoexame e principalmente a melhora na qualidade de vida da população com 60 anos ou mais. Entretanto, não existe restrição de acesso às faixas etárias abaixo desta idade, porém não são registrados para fins de cobertura da ação.

Atualmente, há uma população de 216.745 habitantes no município, segundo o Instituto Brasileiro de Geografia e Estatística ${ }^{14}$, sendo que destes aproximadamente 11\% são idosos (> 60 anos).

A rede de atenção básica está constituída por 45 unidades de saúde, com 12 unidades tradicionais de atenção e 33 equipes da Estratégia Saúde da Família, além de 3 pronto-atendimentos.

$\mathrm{Na}$ atenção secundária o Centro de Especialidades Odontológicas (CEO) conta com uma estomatologista que é responsável pela retriagem dos pacientes com suspeita de lesões potencialmente cancerizáveis identificadas.

A ação é estruturada a partir de uma reunião da coordenação de saúde bucal da Secretaria Municipal da Saúde, com os cirurgiões-dentistas 
da rede básica, que tem por objetivo que as próprias equipes de saúde proponham estratégias de acordo com o contexto local, propiciando a troca de experiências desenvolvidas.

Além da pactuação das metas a serem alcançadas, promove-se a padronização para o registro das informações, bem como planejamento coletivo ascendente e democrático das ações a serem efetivadas.

Diversas estratégias para a adesão dos idosos surgem desse planejamento, como os adesivos que identificam os que já realizaram o exame bucal, a entrega de espelhos de bolso para os usuários realizarem o autoexame, a confecção de camisetas e folders educativos, além da implantação do "Cantinho do autoexame" nas unidades de saúde.

Outro fator fundamental para a ação é a qualificação dos profissionais de saúde $(\mathrm{CD}$, enfermeiras, médicos e fonoaudiólogos), voltada prioritariamente para o reconhecimento de lesões através da utilização de fotografias coloridas das lesões a serem identificadas.

Uma profissional estomatologista realiza a explicitação da mucosa bucal normal, de lesões benignas, de lesões pré-cancerosas e do câncer já presente. São abordadas ainda orientações acerca da importância do autoexame bucal rotineiramente.

Para os dentistas, este momento tem como objetivo principal o auxílio no detalhamento dos exames bucais e reconhecimento das lesões realmente cancerizáveis. Para os demais profissionais o intuito é a sensibilização quanto à importância da ação, despertando um olhar diferenciado para a cavidade bucal.

A ação se inicia no sábado de mobilização nacional para a vacinação contra a gripe para os idosos, e se estende por duas semanas, acompanhando o período destinado a esta atividade, sendo que a agenda do cirurgião-dentista das unidades de saúde fica direcionada para a realização dos exames de prevenção e detecção do câncer.

No período da ação são desenvolvidos procedimentos clínicos de urgência odontológica (02 pacientes/dia) e agendamento de pacientes idosos que realizaram o exame bucal e necessitam de tratamento curativo.

Para as equipes de saúde que atingem suas metas na primeira semana, o profissional CD é remanejado para as unidades que necessitam de suporte, devido ao maior número de idosos em seu território de abrangência.

As unidades recebem um kit contendo luvas de procedimento, gorro, máscara, espátula de madeira, pacotes de gaze, guardanapo de papel, álcool gel e lanternas utilizadas nas VD e na campanha externa, além de materiais educativos (cartazes e panfletos), adesivos com a frase "Já fiz meu exame de boca, e você?" e espelhos de bolso para divulgação do autoexame bucal.

Todos os exames realizados são registrados em planilhas próprias e enviados para a Secretaria Municipal da Saúde e consolidados diariamente pela coordenação de saúde bucal que monitora a evolução da ação e traça novas estratégias para ampliação da cobertura, caso exista a necessidade.

A planilha 01 é utilizada para registrar todos os idosos examinados, abrange informações básicas como o nome do paciente, data do exame, data de nascimento, idade, índice de avaliação profissional (normal-0, alteração reversível-1 e suspeita de lesão cancerizável-2), número de recusas (pacientes que não permitiram a realização do exame bucal) e número de evasões (idosos que fizeram o exame em outra unidade, que não a sua de origem).

$\mathrm{Na}$ planilha 02 constam os pacientes com suspeita de lesões cancerizáveis (índice de avaliação profissional 2), indicados para a segunda avaliação, os quais podem ser agendados no CEO ou atendidos nas próprias unidades de saúde por uma profissional estomatologista que realiza as biópsias, nos casos urgentes ou em casos de dificuldade de locomoção pelo paciente.

Ao formulário de referência e contrarreferência, anexa-se a ficha de localização de lesão, com figuras esquematizadas da face e cavidade bucal. Esta ficha contém dados pessoais do paciente, itens referentes a hábitos (tabagismo e etilismo), uso de próteses, casos de câncer na família e campo destinado a descrição minuciosa da lesão encontrada.

Os pacientes com diagnóstico positivo de câncer bucal são encaminhados para tratamento no Ambulatório de Cabeça e Pescoço do Centro de Oncologia de Marília (COM) ou para o Ambulatório Mário Covas do Hospital de Clínicas em nível de atenção terciária.

Ao término da ação, os dados coletados são enviados para a FOSP, através do preenchimento da planilha 03 (condensação dos dados das planilhas 01 e 02), com a quantidade de pacientes examinados durante a ação (todos os idosos atendidos), quantidade de pacientes que apresentaram alterações reversíveis (próteses mal adaptadas, aftas, dentre outras), quantidade de pacientes que apresentaram lesões cancerizáveis (encaminhados para biopsias) e a quantidade de pa- 
cientes que não compareceram para a $2^{\text {a }}$ avaliação. É enviada, também, a ficha para coleta dos casos encaminhados para biópsia.

Assim foram analisados todos os registros da ação disponibilizados pela coordenação de saúde bucal da Secretaria Municipal da Saúde de Marília no período de 2006 a 2011. Os registros referentes aos anos anteriores não estão disponíveis em nenhum instrumento de avaliação ou relatório de gestão da instituição.

Para análise dos dados correlacionados entre as duas variáveis, cobertura de exame bucal e cobertura de exame bucal na vacinação, foi utilizado o coeficiente de Pearson ao nível de significância de 5\%.

A execução do trabalho teve prévia aprovação junto ao Comitê Municipal de Avaliação e Pesquisa da Secretaria Municipal da Saúde de Marília.

\section{Resultados}

Considerando o planejamento como instrumento de gestão, promotor de desenvolvimento gerencial, exercendo forte influência sobre o compromisso das pessoas com os objetivos institucionais, sendo uma atitude permanente da organização e do administrador ${ }^{15}$, a coordenação de saúde bucal realizou com os CD da rede básica reunião de planejamento coletivo e participativo, utilizando como metodologia o Brainstorming ("tempestade de ideias"), que incentiva a criatividade, iguala o envolvimento dos participantes para a objetividade, economiza tempo e recursos e é de fácil emprego cotidiano.

Os 96 CD da rede básica se dividiram em 07 grupos, de acordo com as regiões do município. Cada grupo levantou as dificuldades em aumentar a cobertura de idosos examinados, refletiram sobre as causas existentes e elaboraram estratégias compatíveis com a prática local.

No decorrer dos anos, durante as reuniões de organização das ações para a campanha foram identificadas diversas dificuldades no processo de trabalho, assim como foram construídas diversas estratégias de enfrentamento (Quadro 1).

O objetivo destas estratégias foi ampliar a cobertura de exames de prevenção e detecção precoce do câncer bucal, além da orientação dos profissionais que compõem as equipes de saúde e ainda a própria população alvo da ação.
Em 2009 a ação registrou 1.292 idosos que não permitiram a realização do exame bucal (recusas), sendo esta a principal dificuldade abordada na reunião de planejamento do ano seguinte. Nesta reunião, os grupos elencaram as causas e construíram as estratégias necessárias para minimizar o número de recusas (Quadro 2).

$\mathrm{Na}$ ação de 2010, foram registradas 1.169 recusas e 829 em 2011, evidenciando um decréscimo em relação ao ano anterior.

O resultado destas estratégias fica evidente na análise do Gráfico 1 que aponta um incremento importante ano a ano na cobertura de exames realizados na população alvo da campanha (> 60 anos) com uma cobertura de $21 \%$ em 2006 para $62 \%$ no ano de 2011.

$\mathrm{Na}$ Tabela 1 o número absoluto de exames bucais realizados durante a ação aumenta ano a ano, sendo que em cinco anos, praticamente triplicou de 4.928 exames no ano de 2006 para 17.986 em 2011.

Portanto é notável a superação do número de doses aplicadas da vacina na população idosa do município, em todos os anos analisados.

Observou-se correlação positiva, significativa, entre ano e porcentagem de cobertura de exame bucal e porcentagem de cobertura de exame bucal na vacinação, ou seja, com o passar dos anos de estudo, aumentou a \% cobertura de exame bucal $(p=0,0009)$ e a $\%$ cobertura de exame bucal na vacinação $(\mathrm{p}=0,0087)$.

A Tabela 2 demonstra os resultados encontrados no presente estudo, numa comparação com os dados de cobertura de exame bucal em municípios com número de habitantes que variam de 200 a 240 mil, semelhantes ao porte populacional de Marília, e que obtiveram resultados de maior cobertura de exames no Estado. As três maiores coberturas de exames foram obtidas por Marília com 62,8\%, seguido por Americana com $8,7 \%$, e Araraquara com 7,0\%.

A Tabela 3 demonstra que a porcentagem de exames bucais realizados nos municípios pertencentes à DRS-IX Marília superam as demais, sendo que dos 34.462 idosos examinados, 17.986 são do município de Marília, mais da metade da cobertura de todos os exames realizados.

O Gráfico 2 demonstra o número de pacientes encaminhados ao CEO para realização da segunda avaliação. Este fluxo é necessário nos casos em que o dentista tem dificuldades em definir a necessidade da biopsia e solicita retriagem por profissional especializado. 
Quadro 1. Dificuldades e estratégias verificadas pelas equipes responsáveis pelo planejamento do processo de organização da ação de prevenção e deteç̧ão precoce do câncer bucal de Marília.

\begin{tabular}{|c|c|}
\hline Dificuldades da equipe & Estratégias das equipes \\
\hline \multirow{3}{*}{$\begin{array}{l}\text { Atingir a meta de } 100 \% \text { de idosos } \\
\text { examinados no sábado de abertura da ação }\end{array}$} & Realização do exame bucal antes da vacina \\
\hline & $\begin{array}{l}\text { Sensibilização e integração de toda a equipe de saúde } \\
\text { em relação à importância da campanha }\end{array}$ \\
\hline & $\begin{array}{l}\text { Remanejamento dos } \mathrm{CD} \text { de unidades com público alvo } \\
\text { reduzido para unidades com maior número de idosos }\end{array}$ \\
\hline \multirow[t]{6}{*}{$\begin{array}{l}\text { Aproximar a cobertura de idosos examinados } \\
\text { à de idosos vacinados }\end{array}$} & $\begin{array}{l}\text { Ampliação do período da campanha para duas semanas } \\
\text { com adaptação da agenda do CD }\end{array}$ \\
\hline & Agendamento dos exames em USF \\
\hline & $\begin{array}{l}\text { Remanejamento dos CD de unidades com público alvo } \\
\text { reduzido para unidades com maior número de idosos }\end{array}$ \\
\hline & $\begin{array}{l}\text { Busca ativa dos idosos (VD) na última semana da } \\
\text { campanha }\end{array}$ \\
\hline & $\begin{array}{l}\text { Extensão da campanha aos locais de grande } \\
\text { movimentação popular (supermercados, feiras livres e } \\
\text { comércio) }\end{array}$ \\
\hline & Implantação da gestão participativa com os CD \\
\hline $\begin{array}{l}\text { Falta de envolvimento dos demais membros } \\
\text { da equipe }\end{array}$ & Sensibilização e integração de toda a equipe de saúde \\
\hline $\begin{array}{l}\text { Tempo dispendido para preenchimento das } \\
\text { planilhas }\end{array}$ & Planilhas de preenchimento mais sucinto \\
\hline $\begin{array}{l}\text { Falta de divulgação na mídia que prioriza a } \\
\text { campanha de vacinação da gripe }\end{array}$ & $\begin{array}{l}\text { Divulgação na mídia local (faixas, rádios, jornais, } \\
\text { material educativo...) }\end{array}$ \\
\hline \multicolumn{2}{|l|}{$\begin{array}{l}\text { Recursos humanos reduzidos em relação à } \\
\text { equipe estruturada para a campanha de } \\
\text { vacinação da gripe }\end{array}$} \\
\hline \multicolumn{2}{|l|}{$\begin{array}{l}\text { Início da vacinação após o acolhimento } \\
\text { médico e odontológico }\end{array}$} \\
\hline \multicolumn{2}{|l|}{$\begin{array}{l}\text { Remanejamento dos CD de unidades com } \\
\text { público alvo reduzido para unidades com } \\
\text { maior número de idosos }\end{array}$} \\
\hline \multicolumn{2}{|l|}{$\begin{array}{l}\text { Participação dos estagiários de odontologia da } \\
\text { universidade }\end{array}$} \\
\hline $\begin{array}{l}\text { Maior tempo para a realização do exame } \\
\text { bucal em relação à aplicação da vacina }\end{array}$ & $\begin{array}{l}\text { Realização do exame bucal antes da aplicação da vacina } \\
\text { da gripe }\end{array}$ \\
\hline \multicolumn{2}{|l|}{$\begin{array}{l}\text { Participação dos estagiários de odontologia da } \\
\text { universidade }\end{array}$} \\
\hline Recusa do paciente por desinformação & $\begin{array}{l}\text { Realização de palestras nas unidades (grupos de } 3^{a} \\
\text { idade) }\end{array}$ \\
\hline \multicolumn{2}{|l|}{$\begin{array}{l}\text { Realizar exame bucal nos acamados de UBS } \\
\text { (VD) }\end{array}$} \\
\hline Área rural extensa & $\begin{array}{l}\text { Remanejamento dos CD substitutos, para que a } \\
\text { unidade não fique desassistida }\end{array}$ \\
\hline
\end{tabular}


Quadro 2. Causas e estratégias verificadas pelas equipes responsáveis pelo planejamento do processo de organização da ação de prevenção e detecção precoce do câncer bucal em Marília.

\begin{tabular}{|c|c|}
\hline Causas das recusas & Estratégias das equipes \\
\hline \multirow[t]{6}{*}{ Falta de informação e divulgação da ação } & $\begin{array}{l}\text { Melhorar a divulgação da campanha na mídia (jornal, } \\
\text { rádio, tv). }\end{array}$ \\
\hline & $\begin{array}{l}\text { Conscientizar a população através de distribuição de } \\
\text { cartazes e panfletos explicativos na unidade, } \\
\text { supermercados, farmácias, padarias, reunião de } \\
\text { comunidade, orientações dos ACS. }\end{array}$ \\
\hline & $\begin{array}{l}\text { Divulgar com antecedência que a campanha da gripe e } \\
\text { câncer de boca será ao mesmo tempo. }\end{array}$ \\
\hline & $\begin{array}{l}\text { Estabelecer parcerias com instituições (igreja, centros } \\
\text { comunitários, grupos da 3a idade, Associação de } \\
\text { Combate ao Câncer). }\end{array}$ \\
\hline & $\begin{array}{l}\text { Sensibilizar todos os membros da equipe quanto à } \\
\text { importância da prevenção do câncer bucal. }\end{array}$ \\
\hline & $\begin{array}{l}\text { Divulgar a campanha, através da distribuição de adesivos } \\
\text { que identificam os idosos examinados. }\end{array}$ \\
\hline \multirow{2}{*}{$\begin{array}{l}\text { Vergonha de remover a prótese e } \\
\text { constrangimento quanto às condições da } \\
\text { mesma (antigas, fraturadas, falta de higiene). }\end{array}$} & $\begin{array}{l}\text { Realizar acolhimento adequado através de abordagem } \\
\text { humanizada, carinhosa, discreta e solidária. }\end{array}$ \\
\hline & Fortalecer o vinculo. \\
\hline \multirow{2}{*}{$\begin{array}{l}\text { Fatores sócio, educacional e cultural (medo } \\
\text { da doença). }\end{array}$} & Mudar a abordagem (CÂNCER - palavra impactante). \\
\hline & Desenvolver o trabalho multiprofissional \\
\hline \multirow{8}{*}{$\begin{array}{l}\text { Desinteresse, desvalorização e falta de } \\
\text { conscientização do paciente em relação à } \\
\text { importância do exame e do diagnostico } \\
\text { precoce }\end{array}$} & $\begin{array}{l}\text { Divulgar na unidade os casos com diagnóstico precoce } \\
\text { resolvidos com sucesso. }\end{array}$ \\
\hline & $\begin{array}{l}\text { Utilizar recursos visuais apelativos com divulgação de } \\
\text { imagens de casos de câncer bucal avançado. }\end{array}$ \\
\hline & $\begin{array}{l}\text { Trabalhar com grupos educativos: trocas de experiências } \\
\text { considerando suas historias de vida. }\end{array}$ \\
\hline & $\begin{array}{l}\text { Estimular o auxílio e interesse da equipe na campanha e } \\
\text { durante o ano para divulgação da importância do exame } \\
\text { preventivo e diagnóstico precoce. }\end{array}$ \\
\hline & $\begin{array}{l}\text { Divulgar a importância da realização do exame junto à } \\
\text { família do idoso. }\end{array}$ \\
\hline & $\begin{array}{l}\text { Valorizar o exame da boca na rotina de atendimento. } \\
\text { Disponibilizar visitas domiciliares antecipadas ao período } \\
\text { da campanha para os pacientes com história de recusa } \\
\text { anterior. }\end{array}$ \\
\hline & $\begin{array}{l}\text { Distribuir informes educativos e brindes (espelhos de } \\
\text { bolso) após a realização do exame. }\end{array}$ \\
\hline & Realizar o cantinho do autoexame nas unidades de saúde. \\
\hline \multicolumn{2}{|l|}{$\begin{array}{l}\text { Alto nível sócio econômico cultural (relatam } \\
\text { que fazem o exame particular) }\end{array}$} \\
\hline $\begin{array}{l}\text { Preconceito para com a qualidade oferecida } \\
\text { pelo serviço público }\end{array}$ & \\
\hline
\end{tabular}




\begin{tabular}{|c|c|c|}
\hline \multirow[t]{8}{*}{ 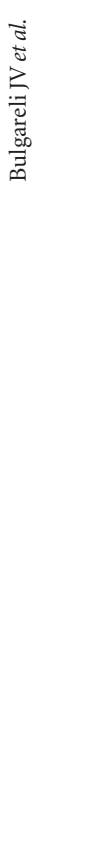 } & Quadro 2. continuação & 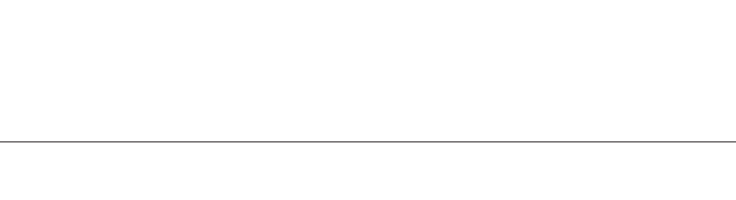 \\
\hline & Causas das recusas & Estratégias das equipes \\
\hline & Parentesco com o C.D & $\begin{array}{l}\text { Desmistificar a imagem negativa do serviço público } \\
\text { valorizando o profissional dentista, através da divulgação } \\
\text { das ações que as unidades de saúde desenvolvem }\end{array}$ \\
\hline & & Integrar os serviços particulares junto à campanha \\
\hline & $\begin{array}{l}\text { Ansiedade do idoso em relação à espera para } \\
\text { ser examinado }\end{array}$ & Local do exame bucal próximo ao da sala de vacina \\
\hline & $\begin{array}{l}\text { Apoio de outros profissionais, } \\
\text { principalmente a equipe de enfermagem. }\end{array}$ & \\
\hline & $\begin{array}{l}\text { Falta de envolvimento entre os profissionais } \\
\text { da equipe. }\end{array}$ & \\
\hline & & $\begin{array}{l}\text { Capacitação da equipe da unidade, para maior } \\
\text { envolvimento durante a campanha e no decorrer do ano, }\end{array}$ \\
\hline
\end{tabular}

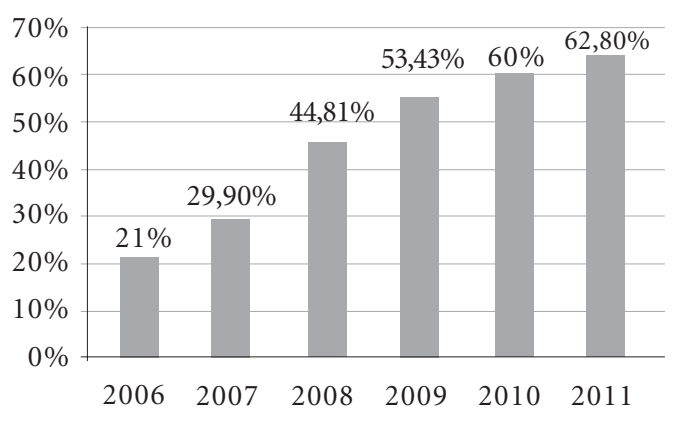

$\%$ cobertura de exames

Gráfico 1. Percentual de cobertura de examinados com idade igual ou superior a 60 anos durante a ação de prevenção e detecção precoce do câncer bucal.

Fonte: SMS Marília/SP

Em 2011 foram reavaliados pelo CEO 311 pacientes, o que corresponde a 1,7\% dos encaminhamentos. Já no ano de 2006 foram encaminhados 329 pacientes para segunda avaliação que corresponde a 6,6\% dos encaminhamentos, praticamente $5,0 \%$ a mais que em 2011, que o número absoluto de exames foi aproximadamente três vezes maior que 2006.

Esta informação pode estar relacionada à melhora da qualidade técnica do cirurgião-dentista no processo de avaliação e identificação das lesões da cavidade bucal.

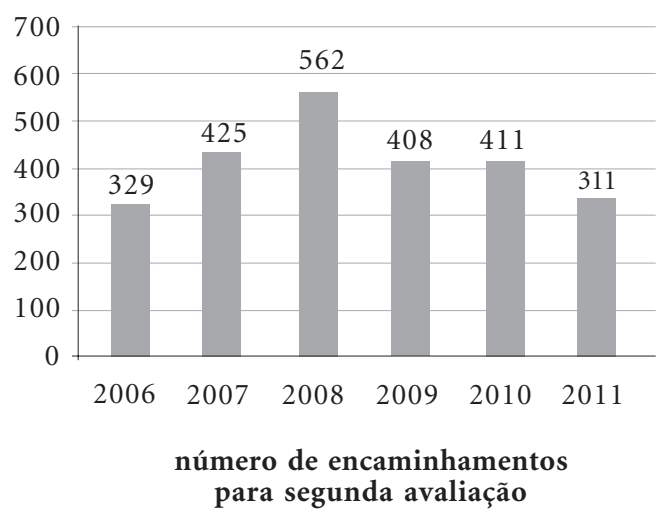

Gráfico 2. Número de pacientes encaminhados para segunda avaliação no Centro de Especialidades Odontológicas (CEO) no período de 2006 a 2011.

Fonte: SMS Marília/SP

\section{Discussão}

Em um cenário em que o aumento do número de casos novos de câncer vem assumindo um papel preocupante e já constitui a segunda causa de morte por doenças no Brasil, atrás apenas das doenças cardiovasculares, este estudo é de grande relevância para a sensibilização dos gestores em saúde pública em relação à gestão compartilhada no planejamento e organização de ações voltadas à prevenção e diagnóstico precoce do câncer bucal. 
Tabela 1. Número absoluto e percentual de exames bucais realizados durante a ação de prevenção e detecção precoce do câncer bucal e número e percentual de doses aplicadas da vacina de gripe no período de 2006 a 2011.

\begin{tabular}{lcccccc}
\hline Ano & $\begin{array}{c}\text { População } \\
\mathbf{2} \mathbf{6 0} \text { anos }\end{array}$ & $\begin{array}{c}\text { No exames } \\
\text { bucais }\end{array}$ & $\begin{array}{c}\text { \% cobertura } \\
\text { *exame bucal }\end{array}$ & $\begin{array}{c}\text { Doses aplicadas } \\
\text { vacina gripe }\end{array}$ & $\begin{array}{c}\text { \% cobertura } \\
\text { vacinação }\end{array}$ & $\begin{array}{c}\text { \% cobertura } \\
\text { *exame bucal } \\
\text { na vacinação }\end{array}$ \\
\hline 2006 & 23.467 & 4.928 & $21,00 \%$ & 18.586 & $79,20 \%$ & $26,51 \%$ \\
2007 & 22.905 & 6.392 & $27,90 \%$ & 17.848 & $74,71 \%$ & $35,81 \%$ \\
2008 & 27.523 & 12.335 & $44,81 \%$ & 18.337 & $66,62 \%$ & $67,26 \%$ \\
2009 & 28.626 & 15.296 & $53,43 \%$ & 19.839 & $69,00 \%$ & $77,10 \%$ \\
2010 & 28.626 & 17.195 & $60,06 \%$ & 20.601 & $71,96 \%$ & $83,46 \%$ \\
2011 & 28.626 & 17.986 & $62,8 \%$ & 22.285 & $77,8 \%$ & $80,70 \%$ \\
\hline
\end{tabular}

Fonte IBGE. * Correlação de Pearson $(\mathrm{p} \leq 0,05)$

Tabela 2. Número absoluto e percentual de exames bucais realizados durante a ação de prevenção e detecção precoce do câncer bucal, em comparação a municípios com população entre 200 a 240 mil habitantes, no ano de 2011.

\begin{tabular}{lccrc}
\hline Municípios & $\begin{array}{c}\text { População } \\
\text { município }\end{array}$ & $\begin{array}{c}\text { População } \\
\geq \mathbf{6 0} \text { anos }\end{array}$ & $\begin{array}{c}\text { No exames } \\
\text { bucais }\end{array}$ & $\begin{array}{c}\text { \% de cobertura } \\
\text { de exames bucais }\end{array}$ \\
\hline Americana & 210.638 & 26.931 & 2.360 & $8,7 \%$ \\
Araraquara & 208.662 & 29.103 & 2.048 & $7,0 \%$ \\
Embu & 240.230 & 16.396 & 593 & $3,6 \%$ \\
Indaiatuba & 201.619 & 20.857 & 1.315 & $6,3 \%$ \\
Marília & 214.742 & 28.626 & 17.986 & $62,8 \%$ \\
Sumaré & 241.311 & 20.204 & 831 & $4,1 \%$ \\
\hline
\end{tabular}

Fonte: FOSP e IBGE

Tabela 3. Número absoluto e percentual de exames bucais realizados durante a ação de prevenção e detecção precoce do câncer bucal, comparando DRS do Estado de São Paulo, no ano de 2011.

\begin{tabular}{lcccc}
\hline $\begin{array}{c}\text { Departamento Regional } \\
\text { de Saúde (DRS) }\end{array}$ & $\begin{array}{c}\text { População } \\
\text { DRS }\end{array}$ & $\begin{array}{c}\text { População } \\
\mathbf{2 6 0} \text { anos }\end{array}$ & $\begin{array}{c}\text { No exames } \\
\text { bucais }\end{array}$ & $\begin{array}{r}\text { \% de cobertura } \\
\text { de exames bucais }\end{array}$ \\
\hline DRS 01 Grande São Paulo & 19.867 .456 & 21.114 .593 & 290.052 & $13,71 \%$ \\
DRS 07 Campinas & 4.098 .799 & 466.744 & 42.916 & $9,1 \%$ \\
DRS 09 Marília & 1.074 .333 & 154.333 & 34.462 & $22,32 \%$ \\
DRS 17 Taubaté & 2.292 .055 & 248.967 & 34.793 & $13,97 \%$ \\
\hline
\end{tabular}

Fonte: FOSP e IBGE

Discute ainda o processo de trabalho das unidades de saúde da rede básica podendo ser reproduzido em outras localidades que realizam a prevenção do câncer bucal.

A valorização da campanha de vacinação contra a gripe, em âmbito nacional, facilitou a implantação da ação de prevenção e detecção preco- ce de câncer bucal no Estado de São Paulo, através do incentivo da FOSP. Desta forma, sua integração junto à campanha de vacinação definiu como prioritária a população de 60 anos ou mais.

A identificação de casos de câncer bucal ainda em fase inicial, para os quais, as intervenções terapêuticas podem ser benéficas aos pacientes, 
o estímulo ao autoexame e, principalmente, possibilitar uma melhora na qualidade de vida destas pessoas são os objetivos principais da ação.

A literatura atribui as principais restrições ao rastreamento de câncer, as quais de uma maneira geral dizem respeito à reduzida proporção de pessoas testadas que são efetivamente beneficiadas, aos interesses comerciais associados, aos efeitos adversos relacionados ao baixo valor preditivo positivo dos recursos diagnósticos e à falta de evidências suficientes de efetividade. Além disso, a implementação do diagnóstico precoce e da terapêutica apropriada não consegue, em muitos casos, evitar a progressão letal da doença ${ }^{16}$.

No que se refere ao câncer bucal, não é possível aplicar esta máxima, pois envolve apenas a realização do exame visual da boca, não havendo necessidade de custos elevados, bem como a associação a exames laboratoriais.

Além disso, mesmo se a busca pelo câncer bucal não fosse realizada, as demais ações como: educação em saúde, exame de próteses e tratamento de lesões em tecidos moles da boca por si só justificam a integração do exame bucal nas campanhas de vacinação.

Como indicador de alta resolutividade do estudo destaca-se os encaminhamentos para elucidação diagnóstica (segunda avaliação), sendo que durante a realização dos exames no ano de 2011 foram identificadas 697 situações que apresentavam lesões reversíveis e 207 suspeitas de malignidade, sendo que, destas, 311 pacientes foram encaminhados para o CEO, os quais foram efetivamente seguidos pela população, que compareceu em $72,35 \%$ das consultas agendadas, havendo a confirmação de dois casos de carcinoma epidermoide, os quais foram adequadamente tratados não havendo evolução de nenhum caso para óbito.

Os dois casos identificados em Marília representam $0,01 \%$ de confirmação diagnóstica da doença em relação aos 17.986 exames realizados no ano de 2011, o que comparado aos municípios de semelhante porte populacional como Sumaré, Indaiatuba e Araraquara, provavelmente aponta para um resultado bastante significativo ao longo dos anos que se sucedem as ações de prevenção e detecção precoce do câncer em Marília. Tal hipótese se alicerça no resultado de casos diagnosticados nos municípios de Sumaré, Indaiatuba e Araraquara que respectivamente realizaram 831, 1.315 e 2.048 exames no ano de 2011, com percentual de identificação dos casos da doença de $0,36 \%, 0,15 \%$ e $0,04 \%$, todos acima do município de Marília. Se realizarmos o soma- tório dos exames realizados por estes três municípios, o número obtido não atinge metade dos exames realizados no município estudado e houve a identificação de seis casos confirmados, ou seja, três vezes mais que o identificado em Marília. Hipoteticamente, isso pode significar que parte desses casos pode ter sido diagnosticada em grau de severidade mais avançado.

Isto pode significar que Marília tem obtido êxito no controle da doença na população através das orientações, durante a ação de prevenção e detecção precoce ao longo do tempo, para a realização do autoexame e identificação de lesões pré-cancerizáveis, além de adoção de hábitos de vida saudáveis e alerta as condições que podem contribuir para o aparecimento do câncer, como não fumar, não beber, não se expor ao sol sem proteção, não relacionados ao fator hereditário, produzindo resultado efetivo no controle do aparecimento do câncer bucal.

Mesmo sabendo-se que a faixa etária de maior incidência do câncer bucal situa-se entre $45 \mathrm{e}$ 55 anos de idade ${ }^{17}$, o foco dos exames durante a ação são voltados para a população igual ou acima de 60 anos.

Esta priorização se deve a orientação da FOSP, devido à associação junto a Campanha Nacional de Vacinação do Idoso, entretanto um processo de integração como este é capaz de divulgar sobremaneira a necessidade do autoexame como prática rotineira de prevenção do câncer bucal para toda a população, além de que qualquer faixa etária pode receber o exame bucal, que não é computado para fins de cobertura populacional durante a ação.

Isto aponta para um impacto ainda maior em relação à realização de exames e educação em saúde bucal no que se refere à prevenção do câncer, sem considerar as ações educativas realizadas extramuro (consultório) nos espaços sociais dos territórios e em grupos existentes nas unidades de saúde.

O mais importante é que as ações de prevenção e diagnóstico precoce acabam se incorporando na rotina de trabalho dos odontólogos na atenção básica, o que também pode explicar o índice de identificação da doença menor com maior número de exames realizados no município de Marília em comparação aos outros municípios de porte populacional semelhante.

Estima-se que a mortalidade por câncer bucal possa ser reduzida pelo esforço em identificar lesões precoces em segmentos de população com risco mais elevado e efetivo tratamento dessas lesões malignas ${ }^{18}$. 
Assim, as ações vivenciadas para a prevenção do câncer bucal e incorporadas no processo de trabalho na atenção básica têm sido o caminho percorrido para modificar o cenário vivenciado nas duas últimas décadas em relação ao controle efetivo da doença.

Se compreendido o processo saúde/doença em toda a sua complexidade, com seus nexos causais diversos e na sua determinação social, com indivíduos e populações apresentando riscos desiguais de adoecer e morrer, se compreende também que sua modificação exige ações e intervenções do setor saúde e aliança com outros setores que elaboram e implementam políticas públicas.

Esta visão rompe com o isolamento do setor saúde e o inclui num outro horizonte político, trabalhando institucionalmente a intersetorialidade e construindo efetivamente políticas públicas eficazes, resolutivas e que de fato atendam às necessidades da população ${ }^{18}$.

Para tanto, fica evidente a notória melhora no impacto na cobertura populacional frente às ações propostas para a realização da ação de prevenção e detecção precoce do câncer bucal após as estratégias adotadas em conjunto entre os profissionais cirurgiões-dentistas das unidades de saúde, a coordenação de saúde bucal e a vigilância epidemiológica em Marília, tendo a cobertura de exames bucais se aproximado a cada ano em relação ao total de idosos vacinados no município de Marília.

Este resultado é extremamente relevante e significativo na comparação de Marília com os outros municípios de porte populacional semelhante, sendo que o somatório do número de exames realizados em 2011 pelos outros cinco municípios utilizados neste estudo representa apenas 39,7\% dos exames realizados por Marília, que obteve uma cobertura de $62,8 \%$ do total de idosos existentes no município. O município de Marília foi responsável por $52,1 \%$ de todos os exames realizados em sua região, DRS IX Marília, que examinou um total de 34.462 exames em 2011.

O mesmo acontece na análise das regiões de saúde, através dos resultados obtidos nos DRS, sendo que o DRS IX Marília obteve cobertura de $22,32 \%$ dos idosos examinados, frente a $13,97 \%$ no DRS 17 Taubaté, 13,71\% do DRS 01 Grande São Paulo e 9,1\% do DRS 07 Campinas.

Estes dados apenas confirmam a relevância das ações desenvolvidas na rede de atenção a saúde pelo município de Marília com grande impacto na prevenção e detecção do câncer bucal.

A construção de uma rede de protagonistas heterogêneos, a partir de diferentes relações for- mais e informais, implica um processo simultâneo de negociação.

Como resultado desse processo por excelência transforma-se os objetivos iniciais de um ator da ação em objetivos definidos coletivamente, a partir das diversas possibilidades e necessidades dos diferentes atores ${ }^{19}$.

Num contexto em que vários projetos de sociedade/atores se encontram em confronto constante, além do reconhecimento do conflito e sua tematização, é preciso fortalecer a capacidade de escuta do outro e de interação e negociação. Por isso, afirmamos que uma concepção pluralista e comunicativa do planejamento apresenta maior aplicabilidade ${ }^{20}$.

Deste modo, as reuniões de planejamento se configuram em espaços coletivos e democráticos, nos quais são elaboradas metas de cobertura individuais de cada unidade de saúde, gerando ações como a adaptação da agenda do dentista no período da realização dos exames.

Essa ação é necessária haja vista o número de profissionais que aplicam a vacina ser significativamente superior aos que realizam o exame bucal, sendo que as unidades com maior quantidade de idosos necessitam de mais profissionais para agilizar a realização dos exames, com menor recusas pelos pacientes devido à necessidade de espera, pois o tempo despendido para a aplicação da vacina é menor quando comparado ao de avaliação bucal.

Alguns autores têm se dedicado à discussão da gestão no campo da saúde e aportam contribuições importantes para a adequação das organizações, no sentido de que estas se tornem mais flexíveis e se adaptem ao movimento de descentralização e à sua democratização, com a participação efetiva de profissionais e usuários na sua condução ${ }^{21}$. Desta forma, como um coletivo de sujeitos sociais em ação, os trabalhadores são seres humanos dotados de interesses próprios, de necessidades e de desejos, que produzem relações sociais, na medida em que interagem constantemente com outros sujeitos e podem adquirir capacidade para intervir na sua realidade ${ }^{22}$.

Sem dúvida, existem diversos fatores que fortalecem o impacto positivo deste estudo, entretanto, muito esforço ainda será necessário na qualificação do planejamento coletivo e compartilhado das ações, a fim de fortalecer os pontos mais importantes no processo de rastreamento do câncer bucal como a adequada qualificação dos cirurgiões-dentistas, a eficiência do sistema de referência, com capacidade para atender à demanda encaminhada, e a organização de um sis- 
tema de informações eficiente, mas ao mesmo tempo, simples e capaz de fornecer todos os dados para monitoramento e avaliação dos resultados.

Pode-se concluir que o desenvolvimento de estratégias com participação das equipes de saúde e coordenação da vigilância epidemiológica, através de consensos coletivos pactuados entre os sujeitos envolvidos nas ações de prevenção do câncer bucal repercute no aumento de cobertura populacional, comprovado pelos resultados alcançados ao longo dos anos.
Garante ainda que as lesões malignas de câncer bucal sejam diagnosticadas precocemente e tratadas sob a ótica de um prognóstico mais favorável, sendo este estudo, ferramenta extremamente relevante que pode ser reproduzida em outras localidades que se preocupam em garantir a saúde bucal dos idosos.

Faz-se necessário durante o decorrer do ano, a extensão dessas ações para os grupos considerados de maior risco a desenvolver a doença e não contemplados neste estudo.

\section{Colaboradores}

JV Bulgareli, OCCF Diniz, ET Faria, FL Vazquez, KL Cortellazzi e AC Pereira participaram igualmente de todas as etapas de elaboração do artigo. 


\section{Referências}

1. Camargo TC. O ex-sistir feminino enfrentando a quimioterapia para o câncer de mama: um estudo de enfermagem na ótica de Martin Heidegger [tese]. Rio de Janeiro: Universidade Federal do Rio de Janeiro; 2000.

2. Instituto Nacional do Câncer (INCA). O que é o câncer? [página na Internet]. [acessado 2011 jul 5]. Disponível em: http://www1.inca.gov.br/conteudo_ view.asp?id=322

3. World Health Organization (WHO). National cancer control programmes: policies and managerial guidelines. $2^{\text {nd }}$ Edition. Geneva: WHO; 2002.

4. Bittencourt R, Scaletzky A, Boehl J. Perfil epidemiológico do câncer na rede pública de Porto Alegre - RS. Rev. Brasileira de Cancerologia 2004; 50(2):95101.

5. Instituto Nacional de Câncer (INCA). Estimativa da Incidência de Câncer no Brasil em 2010. Brasília: INCA; 2010. [página na Internet]. [acessado 2010 set]. Disponível em: http://www.inca.gov.br/estimativa/ 2010/index.asp?link=tabelaestados.asp\&UF=BR

6. Neville DDS, Damm DDS, Allen MSD, Bouquot MSD. Patologia oral e maxilofacial. Philadelphia: Saunders Company; 1995.

7. Güneri P, Cankaya H, Yavuzer A, Güneri EA, Erisen L, Ozkul D, El SN, Karakaya S, Arican A, Boyacioðlu H. Primary oral cancer in a Turkish population sample: association with sociodemographic features, smoking, alcohol, diet and dentition. Oral Oncol 2005; 41(10):1005-1012.

8. Takezaki T, Hirose K, Inoue M, Hamajima N, Kuroishi T, Nakamura S, Koshikawa T, Matsuura H, Tajima K. Tobacco, alcohol and dietary factors associated with the risk of oral cancer among Japanese. Jpn J Cancer Res 1996; 87(6):555-562.

9. Tavani A, Gallus S, La Vecchia C, Talamini R, Barbone F, Herrero R, Franceschi S. Diet and risk of oral and pharyngeal cancer. An Italian case-control study. Eur J Cancer Prev 2001; 10(2):191-195.

10. Gomes R, Rebello LEFS, Araújo FC, Nascimento E F. A prevenção do câncer de próstata: uma revisão da literatura. Cien Saude Colet 2008; 13(1):235-246.

11. Instituto Nacional de Câncer (INCA). Câncer no Brasil. Dados de Registros de Base Populacional. Brasília: INCA; 2010. [página na Internet]. 2010 [acessado 2010 jul 20]. Disponível em: http:// www.inca.gov.br/regpop/2003/index.asp?link= conteudo_view.asp\&ID=13

12. Thomaz EBAF, Cutrim MCFN, Lopes FF. A importância da educação como estratégia para prevenção e diagnóstico precoce do câncer oral. Rev Acta Oncológica Brasileira 2000; 20(4):149-152.

13. Fundação Oncocentro de São Paulo (FOSP). Relatório da Campanha de Prevenção e Diagnóstico Precoce do Câncer Bucal. São Paulo: FOSP; 2008. [página na Internet]. [acessado 2010 jun]. Disponível em: http://www.fosp.saude.sp.gov.br/html/ fr_sbucal_2005_2008.html
14. Instituto Brasileiro de Geografia e Estatística (IBGE). Censo 2010. [dados na Internet]. [acessado 2013 set 19]. Disponível em: http://www.ibge.gov.br/cidadesat/xtras/perfil.php? $\operatorname{codmun}=352900 \&$ search $=$ saopaulo|marilia

15. Tancredi FB, Barrios SRL, Ferreira JHG. Planejamento em Saúde - Saúde e Cidadania para gestores municipais de serviços de saúde. São Paulo: Fundação Petrópolis; 1998.

16. Borges FT, Garbin CAS, Carvalhosa AA, Castro PHS, Hidalgo LRC. Epidemiologia do câncer de boca em laboratório público do estado de Mato Grosso, Brasil. Cad Saude Publica 2008; 24(9):1977-1982.

17. Eluf-Neto J, Wünsch-Filho V. Screening faz bem à saúde? Rev Ass Med Brasil 2000; 46(4):310-311.

18. Warnakulasuriya KA, Johnson NW. Strengths and weaknesses of screening programmes for oral malignancies and potentially malignant lesions. Eur J Cancer Prev 1996; 5(2):93-98.

19. Machado JMH, Porto MFS. Promoção da saúde e intersetorialidade: a experiência da vigilância em saúde do trabalhador na construção de redes. Epidemiol Serv Saúde [periódico na Internet]. 2003 Set [acessado 2010 ago 15]; 12(3):121-130. Disponível em: http://scielo.iec.pa.gov.br/scielo.php?script= sci_arttext\&pid=S167949742003000300002\&lng=pt.

20. Rivera FJR, Artmann E. Planejamento e gestão em saúde: histórico e tendências com base numa visão comunicativa. Cien Saude Colet 2010; 15(5):22652274.

21. Kopf AW, Hortale VA. Contribuição dos sistemas de gestão de Carlos Matus para uma gestão comunicativa. Cien Saude Colet 2005: 10(Supl.):157-165.

22. Spagnol CA. (Re)pensando a gerência em enfermagem a partir de conceitos utilizados no campo da Saúde Coletiva. Cien Saude Colet 2005; 10(1):119127.

Artigo apresentado em 22/08/2012

Aprovado em 19/10/2012

Versão final apresentada em 31/10/2012 